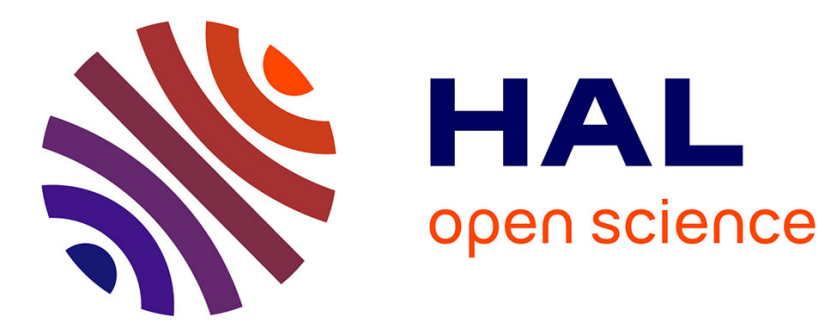

\title{
Adsorption of Polymers with Various Architectures: Mean Field Theory
}

\author{
J. Joanny, A. Johner
}

\section{To cite this version:}

J. Joanny, A. Johner. Adsorption of Polymers with Various Architectures: Mean Field Theory. Journal de Physique II, 1996, 6 (4), pp.511-527. 10.1051/jp2:1996195 . jpa-00248313

\section{HAL Id: jpa-00248313 https://hal.science/jpa-00248313}

Submitted on 1 Jan 1996

HAL is a multi-disciplinary open access archive for the deposit and dissemination of scientific research documents, whether they are published or not. The documents may come from teaching and research institutions in France or abroad, or from public or private research centers.
L'archive ouverte pluridisciplinaire HAL, est destinée au dépôt et à la diffusion de documents scientifiques de niveau recherche, publiés ou non, émanant des établissements d'enseignement et de recherche français ou étrangers, des laboratoires publics ou privés. 


\title{
Adsorption of Polymers with Various Architectures: Mean Field Theory
}

\author{
J.F. Joanny $\left(^{*}\right)$ and A. Johner \\ Institut Charles Sadron, 6 rue Boussingault, 67083 Strasbourg Cedex, France
}

(Received 2 November 1995, received in final form and accepted 4 January 1996)

PACS.82.65.Dp - Thermodynamics of surfaces and interfaces
PACS.68.10.-m - Fluid surfaces and fluid-fluid interfaces
PACS.61.25.Hq - Macromolecular and polymer solutions; polymer melts; swelling

\begin{abstract}
This paper discusses within the framework of a mean field theory the adsorption from a very dilute solution of polymers with various architectures: ring polymers, comblike and starlike polymers and diblock copolymers made of one long adsorbing block and one short nonadsorbing block. By changing the architecture, the relative importance of loops and tails in the adsorbed layer is modified. Ring polymers form only loops in the adsorbed layers and do not form tails. The adsorption can be described by the standard ground state dominance approximation. For all the other architectures, the concentration profile inside the adsorbed layer is the same as for the adsorption of a linear homopolymer with an effective number of monomers ( $2 N$ for stars where each arm contains $N$ monomers and $2 N /(k+1)$ for a comb having $N$ monomers and $(k-1)$ branches). The thickness of the adsorbed layer is also calculated in all cases.
\end{abstract}

\section{Introduction}

Thick polymer layers can be formed at an interface by adsorbing long polymer chains from solution $[1,2]$. The adsorption is in general at least partly irreversible and a large fraction of the adsorbed polymers remain attached to the interface even when the polymer solution is washed. These thick layers have important technological applications in various areas such as colloid stabilisation, adhesion or lubrication.

The structure of adsorbed polymer layers in solution is classically described in terms of loops, tails and trains [1]. The loops are chain segments starting from a monomer in contact with the adsorbing surface and folding back to the surface. The tails are the end segments of the chains that start from an adsorbed monomer and do not fold back on the surface. The trains are chain segments where all the monomers are in contact with the surface, we will not consider them further as they can be considered as a succession of small loops with a size of the order of the monomer size. The relative importance of loops and tails in an adsorbed polymer layer has been elucidated and emphasized by the extensive work initiated by Scheutjens and Fleer [3]. These authors study the statistics of a polymer chain in an adorbed layer in a self-consistent mean field model. The potential seen by each monomer in the adsorbed layer is an average potential written as the sum of the surface attraction and of the mean field excluded volume interaction

(*) Author for correspondence 
due to the other monomers. The monomer concentration profile is calculated numerically and the contributions of loops and tails can then be separated. Close to the adsorbing surface, the concentration is dominated by monomers belonging to loops, the concentration of monomers belonging to loops decreases and the concentration of monomers belonging to tails increases. In the outer part of the adsorbed layer, the polymer concentration is dominated by monomers belonging to tails. Both concentrations of monomers belonging to loops and tails decrease in this region. The end points of the chains are thus expelled from the adsorbing surface. In the crossover between the loop and tail dominated regions, the concentration of monomers belonging to tails has a maximum. This maximum occurs at a distance from the adsorbing surface much smaller than the overall thickness of the layer and thus over most of the adsorbed layer, the monomers belong to the tail segments of the chains.

Another approach to the structure of adsorbed polymer layers based on scaling arguments has been suggested by de Gennes [4]. The detailed statistics of the chains in terms of tails and loops is ignored and only global quantities such as the total concentration are studied. The picture that emerges from the scaling theory is the so-called self-similar grid construction that describes the adsorbed polymer layer locally as a semi-dilute solution with a mesh size increasing linearly from the adsorbing surface. This description accounts rather well for many of the experimentally measured properties of adsorbed polymer solutions (surface excess, concentration profile, hydrodynamic thickness...).

Recently, using both scaling arguments [5] and a mean field theory [6] we have shown that the two approaches are in fact compatible and that the scaling theory can be extended to take into account the differences between tails and loops. This leads to a description in qualitative agreement with the numerical self-consistent field theory and gives scaling predictions for the tail and loop size distributions or the crossover length between the regions dominated by loops and tails. The new mean field approach introduces two order parameters, one associated with loops and the other one with tails. It is valid only in the asymptotic limit where the molecular weight is very large. A detailed comparison with the numerical theory of Scheutjens and Fleer [7] gives however a quantitative agreement in most cases.

In this paper we would like to come back on the roles of loops and tails in adsorbed polymer layers and study some parameters linked to the architecture of the polymer that modify their relative importance: ring polymers do not have any tails and form adsorbed layers containing only loops; branched polymers such as stars or combs have more than two end points per macromolecule and form adsorbed layers with a larger number of tails. Another example is that of a diblock copolymer comprising a long adsorbing block and a short non adsorbing block repelled by the interface; the junction point of the copolymer (that plays the role of one of the end points) is then strongly repelled from the surface. We study all these problems by extending the analytical mean field theory introduced recently to study the adsorption of linear polymers. This type of theory ignores the excluded volume correlations [8] and can thus only be compared qualitatively to experimental results. It is however easy to include in this approach the effect of the polymer architecture which is hardly captured by the scaling theory. We also limit our discussion to adsorption from a very dilute solution.

The paper is organised as follows. In the next section, we discuss the adsorption of ring polymers. In Section 3, we consider starlike and comblike polymers and in Section 4, copolymer adsorption. The last section presents some concluding remarks.

\section{Adsorption of Ring Polymers}

In this section, we study the adsorption onto a solid surface of a very dilute solution of ring polymers of concentration $c_{\mathrm{b}}$ using a mean field theory. This problem has been studied within 
the framework of the Scheutjens-Fleer self-consistent field theory by van Lent et al. [9]. In the adsorbed layer each monomer sees a mean field potential $U(z)$ which is the sum of the excluded volume interaction with the other monomers and of the surface attraction

$$
U(z)=v\left[c(z)-c_{\mathrm{b}}\right]-\frac{a^{2}}{6 d} \delta(z)
$$

The potential is written here in units of $k T$. The excluded volume interaction is proportional to the local concentration $c(z)$; in a good solvent, the excluded volume parameter $v$ is positive. When we consider adsorption from a very dilute solution, the bulk contribution to the molecular potential $v c_{\mathrm{b}}$ is negligible. The surface attraction is a local potential which is modelled by a Dirac $\delta$ function; the precise form of this potential has no influence on the structure of the adsorbed layer at a mesoscopic length scale. In general we have in mind an energy gain of order $k T$ for each monomer in contact with the adsorbing surface and the strength of the attraction is characterised by the adsorption length $d$ which is of the order of the monomer size $a$.

In the mean field theory [8] one first fixes the potential and determines the conformation of one chain in this potential. The molecular potential $U(z)$ is then determined self-consistently. The conformation of a ring polymer is derived directly from that of a linear chain. If $G_{N}\left(z, z^{\prime}\right)$ is the propagator of a linear chain (in the same molecular potential $U$ ), the local concentration of ring polymer in the adsorbed layer $c(z)$ is proportional to $G_{N}(z, z)$.

The propagator of a linear chain in the potential $U(z)$ satisfies a Schrödinger-like equation [10]

$$
-\frac{\partial G_{n}}{\partial n}=-\frac{a^{2}}{6} \frac{\partial^{2} G_{n}}{\partial z^{2}}+U(z) G_{n}
$$

In the polymer adsorption problem, this equation has a bound state of energy $-\varepsilon$ and can be solved using the so-called ground state dominance approximation. The ground state eigenfunction is obtained from the eigenvalue equation

$$
-\varepsilon \psi_{0}=-\frac{a^{2}}{6} \frac{\partial^{2} \psi_{0}}{\partial z^{2}}+U(z) \psi_{0}
$$

Using a proper normalisation, in the ground state dominance approximation, the local ring polymer concentration, $c(z)$ is equal to $c(z)=\psi_{0}(z)^{2}$. If the solution is so dilute that the bulk concentration can be ignored $\psi_{0}(z)$ vanishes at infinity and

$$
\psi_{0}(z)=\frac{(2 \varepsilon / v)^{1 / 2}}{\sinh (y+b)}
$$

We have here introduced the length scale $\lambda=\left(a^{2} / 6 \varepsilon\right)^{1 / 2}$ and defined a dimensionless distance $y=z / \lambda$. The binding energy $\varepsilon$ is shown below to be of order $1 / N$ with a large prefactor that varies logarithmically with the bulk concentration. The length scale $\lambda$ is thus proportional to the Gaussian radius of gyration of the chains but it is somehow smaller because of this prefactor. The integration constant $b \ll 1$ is fixed by the strength of the attraction of the monomers to the wall: $\operatorname{coth} b=\lambda / d$. The adsorption length $d=\lambda b$ is of the order of a monomer size when the energy gained per monomer in contact with the surface is of order $k T$. In the intermediate range, $d<z<\lambda$ the concentration profile given by (4) is the standard mean field scaling profile $c(z)=a^{2} /\left(3 v z^{2}\right)$; at larger distances, the concentration decays exponentially over a length $\lambda / 2$.

Following the ideas of reference [11], we now calculate the propagator of a linear chain in the molecular potential due to the adsorbed ring polymers by solving equation (3) with a concentration profile given by the ground state dominance approximation $(4): c(z)=2 \varepsilon /\left[v \sinh ^{2}(y+b)\right]$. 
We define the number of blobs per chain $s=n \varepsilon$ and use Laplace transformation with respect to the variable $s: \tilde{G}_{p}=\int_{0}^{\infty} \mathrm{d} s G_{s} \mathrm{e}^{-p s}$ The Laplace transform of the propagator is equal to

$$
\begin{aligned}
\tilde{G}_{p}\left(y, y^{\prime}\right)= & -\frac{1}{2 \omega\left(\omega^{2}-1\right)}\left\{-\mathrm{e}^{-\omega\left(y+y^{\prime}\right)}[\operatorname{coth}(y+b)+\omega]\left[\operatorname{coth}\left(y^{\prime}+b\right)+\omega\right]\right. \\
& \left.+\mathrm{e}^{-\omega\left|y-y^{\prime}\right|}\left[\operatorname{coth}\left(y_{>}+b\right)+\omega\right]\left[\operatorname{coth}\left(y_{<}+b\right)-\omega\right]\right\}
\end{aligned}
$$

where $\omega^{2}=p, y_{>}=\max \left(y, y^{\prime}\right)$ and $y_{<}=\min \left(y, y^{\prime}\right)$. The propagator has been normalised in such a way that $G_{0}\left(y, y^{\prime}\right)=\delta\left(y-y^{\prime}\right)$.

The ring polymer concentration is obtained directly by Laplace inversion of $\tilde{G}_{p}(y, y)$. This function has two singularities, a pole at $p=1$ and a branch cut. We focus here on the limit where the bulk concentration is very low and where $\varepsilon \gg 1 / N$. The contribution of the branch cut can then in a first approximation [12] be obtained by taking the limit $\omega \rightarrow 0$. The normalisation is imposed by the concentration at infinity $c_{b}$.

$$
c(z)=c_{\mathrm{b}}\left\{\operatorname{coth}^{2}(y+b)\left(1-\mathrm{e}^{-y^{2} / s}\right)+(\pi s)^{1 / 2}\left(\mathrm{e}^{2 b}-1\right) \mathrm{e}^{s} \frac{1}{\sinh ^{2}(y+b)}\right\}
$$

The second term on the right hand side, associated to the ground state, is the contribution of the adsorbed chains, the first term is associated with the contribution of the non adsorbed chains that do not have any contact with the adsorbing surface, it relaxes towards the imposed bulk concentration over a distance of the order of the bulk radius of gyration of the ring [13] $R_{\mathrm{g}}=\left(N a^{2} / 12\right)^{1 / 2}$ much larger than the adsorption layer thickness $\lambda$.

The important point is that the iterative procedure that we use is consistent. The correction to the ground state dominance approximation is small over all the adsorbed layer $(y<1)$. The same iterative method cannot be used to study the adsorption of linear polymers, over most of the adsorbed layer, in the region dominated by the tails, the correction to the ground state dominance approximation is large [6].

The binding energy $\varepsilon$ is found by imposing that close to the adsorbing surface, the concentration is the same as found from the ground state dominance approximation $c(z)=$ $2 \varepsilon /\left[v \sinh ^{2}(y+b)\right]$. This gives

$$
\varepsilon=-\frac{1}{N} \log \left\{\frac{c_{\mathrm{b}} v}{2 \varepsilon}(\pi N \varepsilon)^{1 / 2}\left(\mathrm{e}^{2 b}-1\right)\right\} \sim-\frac{1}{N} \log \left\{c_{b} v N^{1 / 2}\right\}
$$

Note that our approximations require $s=N \varepsilon \gg 1$ or that $-\log \left\{c_{\mathrm{b}} v N^{1 / 2}\right\} \gg 1$.

The size $\lambda \sim \varepsilon^{-1 / 2}$ of the adsorbed layer is thus as announced proportional to the radius of gyration $R_{\mathrm{g}}$ but it is much smaller than $R_{\mathrm{g}}$ if the bulk concentration $c_{\mathrm{b}}$ is small enough.

Another interesting feature of the concentration profile given by equation (6) is that this profile is non monotonic, it has a minimum at a distance from the wall proportional to $\lambda$ and then a very shallow maximum. As found for the adsorption of linear chains in reference [6], this minimum is due to the depletion of non adsorbed ring polymers at the edge of the adsorbed layer where they are repelled by the adsorbed polymers.

Finally, it is interesting to compare the adsorption of ring polymers to the adsorption of linear polymers of the same molecular weight at the same concentration obtained in reference [6] and summarised in the next section. In the vicinity of the adsorbing surface, the monomer concentration has the same scaling behavior, the structure of the small loops is identical for rings and linear chains. In the outer region, the adsorbed layer of linear polymers is dominated by the tails and the concentration is much higher than the loop concentration of the ring 
polymer (by a factor 10 in the mean field theory). The binding energy of the linear polymer varies as $\varepsilon=-(1 / N) \log \left\{c_{\mathrm{b}} v\right\}$, it is thus larger than that of the ring polymer. The adsorbed layer thickness for linear polymers is $\lambda=\left(a^{2} / 6 \varepsilon\right)^{1 / 2}$; for ring polymers the adsorbed layer thickness is $\lambda / 2=(1 / 2)\left(a^{2} / 6 \varepsilon\right)^{1 / 2}$. In both cases, at very low concentrations, the binding energy behaves as $\varepsilon=-(1 / N) \log \left\{c_{\mathrm{b}} v\right\}$, the thickness of the adsorbed layer is thus larger for the linear polymer. The adsorbed amount of polymer (adsorbance) $\Gamma$ is in both cases dominated by the small loops; for linear polymers, there are two corrections to this dominant value, a positive correction due to the tails that dominates in the asymptotic limit of very large molecular weights and a negative correction due to the finite size of the layer. For the ring polymers, the tail correction does not exist and the finite size correction can be calculated directly from the ground state dominance approximation $\Gamma=(2 \varepsilon \lambda / v)(\operatorname{coth} b-1)=$ $a^{2} /(3 v d)-\left(2 a \varepsilon^{1 / 2}\right) /\left(v 6^{1 / 2}\right)$. Comparing this result with the adsorbance of a linear chain obtained in reference [6] we find that the finite size correction is smaller for the ring polymer. In the asymtotic limit of infinite molecular weight, we thus expect a larger adsorbance for the linear chain, but in most realistic cases, the adsorbance of the ring polymer is larger because of the finite size correction.

\section{Adsorption of Starlike and Comblike Polymers}

In this section, we study the adsorption of polymers with a branched structure. We first summarize the mean field theory with two order parameters [6] that takes into account both tails and loops in an adsorbed layer. We then discuss the adsorption of starlike and comblike polymers.

\subsection{Adsorption of Gaussian Polymers}

3.1.1. Partition Functions. - The starting point of the mean field theory is the same as for ring polymers. We first impose a mean field potential $U(z)$ and study the polymer statistics in this potential. The potential $U(z)$ is then calculated self-consistently.

We consider here a branched polymer chain where all the branches are linear chains with Gaussian statistics. In this branched polymer, we select one branch comprising $n$ monomers. The total number of monomers of the other branches is $P$. We characterize the conformation of the total macromolecule by its partition function with the constraint that the end point of the selected branch is at a distance $z$ from the adsorbing surface, $Z_{n}(z)$ (see Fig. 1). The selected branch follows Gaussian statistics in the external potential $U$ and the partition function $Z_{n}(z)$ satisfies a Schrödinger-like equation similar to equation (2)

$$
-\frac{\partial Z_{n}}{\partial n}=-\frac{a^{2}}{6} \frac{\partial^{2} Z_{n}}{\partial z^{2}}+U(z) Z_{n}
$$

As discussed above for the ring polymer, the partition function can be written as an expansion in the eigenfunctions of equation (8) and in the limit of very high molecular weights the expansion is dominated by the ground state with an energy $-\varepsilon$. It is useful to introduce the partition function $Z_{n}^{\prime}=\mathrm{e}^{-\varepsilon(n+P)} Z_{n}$. We then introduce the Laplace transform with respect to $n, \tilde{Z}_{p}=\int_{0}^{\infty} \mathrm{d} n Z_{n}^{\prime} \mathrm{e}^{-p n}$ and take the Laplace transform of equation (8)

$$
-p \tilde{Z}_{p}+Z_{n=0}^{\prime}=-\frac{a^{2}}{6} \frac{\partial^{2} \tilde{Z}_{p}}{\partial z^{2}}+U(z) \tilde{Z}_{p}+\varepsilon_{p} \tilde{Z}_{p}
$$

In the following, we always focus on the limit where the number of monomers in the branch $n$ is large. This corresponds to the limit $p \rightarrow 0$ in equation (9). If the typical length scale of 


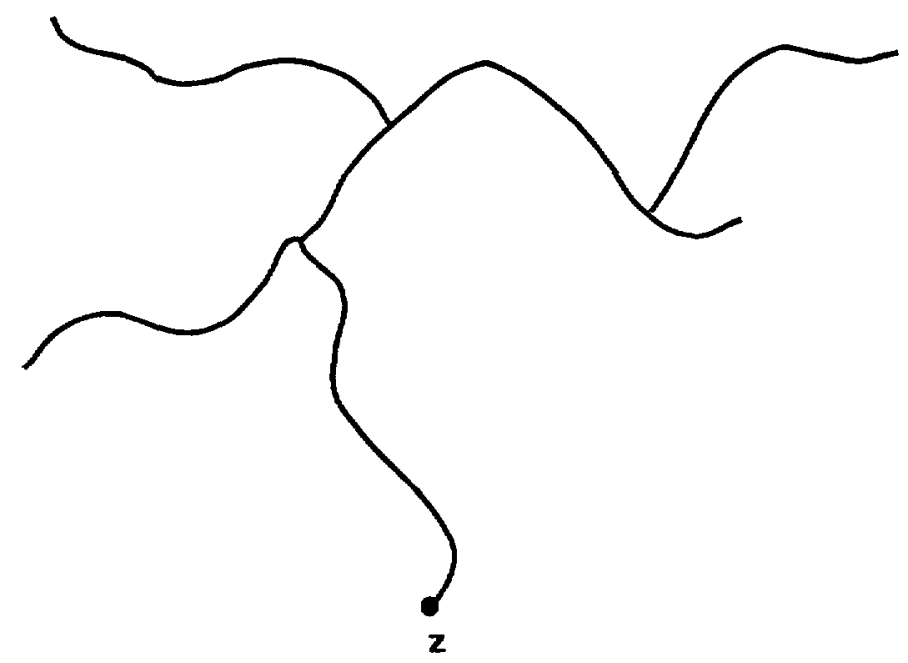

Fig. 1, - A branched polymer with one end point at position $z$.

variation of the partition function $\tilde{Z}_{p}$ is smaller than the Gaussian radius of gyration $R_{n} \sim$ $\left(n a^{2} / 6\right)^{1 / 2}$, the first term in the left hand side of equation $(9), p \tilde{Z}_{p}$, is smaller than the first term on the left hand side $-\left(a^{2} / 6\right) \partial^{2} \tilde{Z}_{p} / \partial z^{2}$ and can be neglected. This requires in particular that the thickness of the adsorbed layer $\lambda \sim a / \varepsilon^{1 / 2}$ be smaller than the radius of gyration of each branch of the polymer. This condition is satisfied only at very low concentration when $\varepsilon N \gg 1$.

The next step is to separate the partition function $Z_{n}^{\prime}$ into a contribution due to adsorbed polymers which have at least one monomer in contact with the adsorbing surface and a contribution due to free or non adsorbed chains where none of the monomers is in contact with the adsorbing surface: $Z_{n}^{\prime}(z)=Z_{n}^{\mathrm{a}}(z)+Z_{n}^{\mathrm{f}}(z)$. The partition function $Z_{n}^{\mathrm{f}}(z)$ vanishes on the surface when $z=0$. The Laplace transforms $\tilde{Z}_{p}^{\mathrm{a}}$ and $\tilde{Z}_{p}^{\mathrm{f}}$ of the partition functions $Z_{n}^{\mathrm{a}}(z)$ and $Z_{n}^{\mathrm{f}}(z)$ are both solutions of equation (9) where the first term on the left hand side can be neglected.

$$
\begin{aligned}
& Z_{n=0}^{\mathrm{a}}=-\frac{a^{2}}{6} \frac{\partial^{2} \tilde{Z}_{\mathrm{p}}^{\mathrm{a}}}{\partial z^{2}}+U(z) \tilde{Z}_{p}^{\mathrm{a}}+\varepsilon \tilde{Z}_{p}^{\mathrm{a}} \\
& Z_{n=0}^{\mathrm{f}}=-\frac{a^{2}}{6} \frac{\partial^{2} \tilde{Z}_{\mathrm{p}}^{\mathrm{f}}}{\partial z^{2}}+U(z) \tilde{Z}_{p}^{\mathrm{f}}+\varepsilon \tilde{Z}_{p}^{\mathrm{f}}
\end{aligned}
$$

3.1.2. Linear Chain Adsorption. - We now recall the essential results obtained in reference [6] for the adsorption of linear chains using this approach. In the limit where the number of monomers is very large, the partition function of the adsorbed chains $Z_{n}^{\mathrm{a}}(z)$ is dominated by the ground state and is independent of the molecular weight $n$, we write $Z_{n}^{\text {a }}(z)=\psi(z)$. In the limit where the number of monomer tends to zero $Z_{n=0}^{\mathrm{a}}=0$. The partition function of the free chains $Z_{n}^{\mathrm{f}}(z)$ is proportional to $\mathrm{e}^{-\varepsilon n}$, the Laplace transform $\tilde{Z}_{p=0}^{\mathrm{f}}$ is thus finite, we define $\phi(z)=\tilde{Z}_{p=0}^{\mathrm{f}}(z)$. In the limit where the number of monomers vanishes, $Z_{n=0}^{\mathrm{f}}=1$. The two order parameters $\psi$ and $\phi$ satisfy thus the equations 


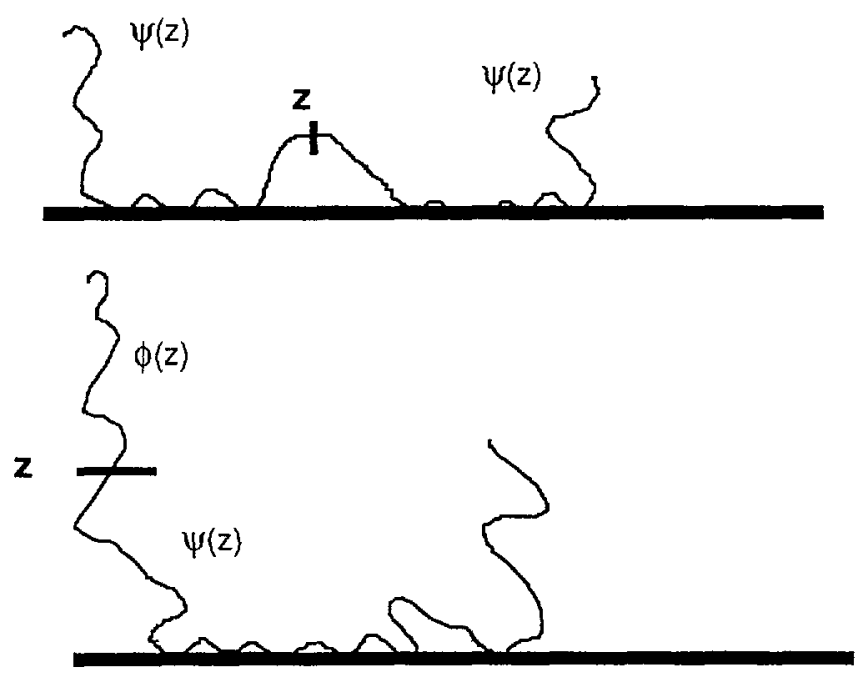

Fig. 2. - Order parameters for linear chain adsorption: $\phi(z)$ describes a tail dangling beyond $z$, $\psi(z)$ describes an adsorbed chain with an end point at position $z$. The loop monomer concentration carries a factor $\psi(z)^{2}$ (see top) whereas the tail monomer concentration carries a factor $\psi(z) \phi(z)$ (see bottom).

$$
\begin{aligned}
& 0=-\frac{a^{2}}{6} \frac{\partial^{2} \psi}{\partial z^{2}}+U(z) \psi+\varepsilon \psi \\
& 1=-\frac{a^{2}}{6} \frac{\partial^{2} \phi}{\partial z^{2}}+U(z) \phi+\varepsilon \phi
\end{aligned}
$$

The monomer concentration in the adsorbed layer can be calculated from the order parameters as (see Fig. 2)

$$
v c(z)=\alpha\left\{\psi^{2}(z)+\frac{2}{N} \psi \phi(z)\right\}
$$

where $\alpha$ is a proportionality constant related to the partition function of a chain in the adsorbed layer.

The molecular potential is $U(z)=v c(z)$. On the surface, $z=0$, one must also include in this potential the surface attraction; we take here into account this attraction by imposing boundary conditions on the wall $\phi=0$ and $\partial \psi / \partial z=-\psi / d$ where $d$ is the so-called adsorption length already introduced for the ring polymers and inversely proportional to the adsorption strength. Inside the adsorbed layer, the binding energy is negligible and the concentration profile has only one characteristic length scale

$$
z^{*}=\left(\frac{N^{2}}{4 \alpha}\right)^{1 / 6} \frac{a}{6^{1 / 2}}
$$

The concentration profile can then be written in a universal form by rescaling the order parameters

$$
\psi=\left(\frac{6 z^{* 2}}{a^{2}}\right)\left(\frac{2}{N}\right) \tilde{\psi}(y), \quad \phi=\left(\frac{6 z^{* 2}}{a^{2}}\right) \tilde{\phi}(y)
$$

where $y=z / z^{*}$. The concentration is then rescaled as

$$
v c(z)=\frac{a^{2}}{6 z^{* 2}} \tilde{c}(y)
$$


The detailed concentration profile has been calculated in reference [6], we only recall here the asymptotic behaviors.

Close to the surface, in the region dominated by the loops

$$
\tilde{\psi}(y)=\frac{2^{1 / 2}}{y}, \quad \tilde{\phi}(y)=-\frac{1}{3} y^{2} \log y, \quad \tilde{c}(y)=\frac{2}{y^{2}}
$$

In the region dominated by the tails,

$$
\tilde{\psi}(y)=\frac{360}{y^{4}}, \quad \tilde{\phi}(y)=\frac{y^{2}}{18}, \quad \tilde{c}(y)=\frac{20}{y^{2}}
$$

The constant $\alpha$ and the crossover distance $z^{*}$ are caculated by writing the conservation of the end points $\Gamma v=\alpha \int \psi \mathrm{d} z$

$$
\alpha=\frac{a^{2}}{3 d^{2} \log ^{2}\left(z^{*} / d\right)}, z^{*}=\left[\frac{N a^{2} d}{122^{1 / 2}} \log \left(z^{*} / d\right)\right]^{1 / 3}
$$

Inside the adsorbed layer, the concentration of monomers belonging to loops and tails are $v c_{1}(z)=\left(a^{2} / 6 z^{* 2}\right) \tilde{\psi}^{2}(y)$ and $v c_{t}(z)=\left(a^{2} / 6 z^{* 2}\right) \tilde{\psi}(y) \tilde{\phi}(y)$. The concentration of end points is $v c_{\mathrm{e}}=\left(a^{2} / 6 z^{* 2}\right)^{2} \tilde{\psi}(y)$.

The binding energy $\varepsilon$ is calculated by balancing the chemical potential of an adsorbed chain to that of a free chain in the bulk. The partition function of an adsorbed chain is $Z=\int \psi \mathrm{d} z=$ $\Gamma v / \alpha$ and the chemical potential of an adsorbed chain is

$$
\mu / k T=-N \varepsilon+\log \Gamma / N-\log Z=-N \varepsilon+\log (\alpha / N v)
$$

Balancing this chemical potential with the bulk chemical potential $\mu=\log c_{\mathrm{b}} / N$ we obtain the binding energy

$$
\varepsilon=-\frac{1}{N} \log \left(c_{\mathrm{b}} v / \alpha\right)
$$

When the distance from the adsorbing surface is very large, the tail order parameter $\phi$ reaches a constant and the loop order parameter $\psi$ decays exponentially as $\psi \sim \mathrm{e}^{-z / \lambda}$ where the thickness of the adsorbed layer is $\lambda=a /(6 \varepsilon)^{1 / 2}$ The concentration profile in the tail region given by equation $(15), c(z)=10 a^{2} /\left(3 z^{2}\right)$ is thus cut off exponentially at a distance $\lambda$.

3.2. Adsorption of Starlike Polymers. - We consider a star polymer comprising $f$ arms each containing $N$ monomers. Two partition functions are necessary to describe the adsorption from a dilute solution: the partition function of a linear chain of $n$ monomers in the mean field potential created by the adsorbed stars $Z_{n}$ and the partition function of a star where one of the arms has $n$ monomers and all the other arms have $N$ monomers $Z_{n}^{s}$.

The partition function of a linear chain can be treated as for the adsorption of linear chains in the previous section by introducing the two order parameters $\psi$ and $\phi$ which satisfy equation (11) with the relevant molecular potential $U(z)$. The star partition function with the end point of the selected branch at position $z, Z_{n}^{\mathrm{s}}(z)$ is decomposed into a free chain and an adsorbed chain contribution. As for linear chains, we introduce the Laplace transform $\tilde{Z}_{p}^{s}=$ $\int_{0}^{\infty} \mathrm{d} n Z_{n}^{s} \mathrm{e}^{-\varepsilon[n+(f-1) N]} \mathrm{e}^{-p n}$ If the star is not adsorbed, the monomers are not in the bound state of energy $\varepsilon$ and the free star partition function $\tilde{Z}_{p}^{\text {sf }}(z)$ is proportional to $\mathrm{e}^{-\varepsilon[(f-1) N]}$. In the limit where $\varepsilon N \gg 1$ that we consider, this partition function is vanishingly small and 


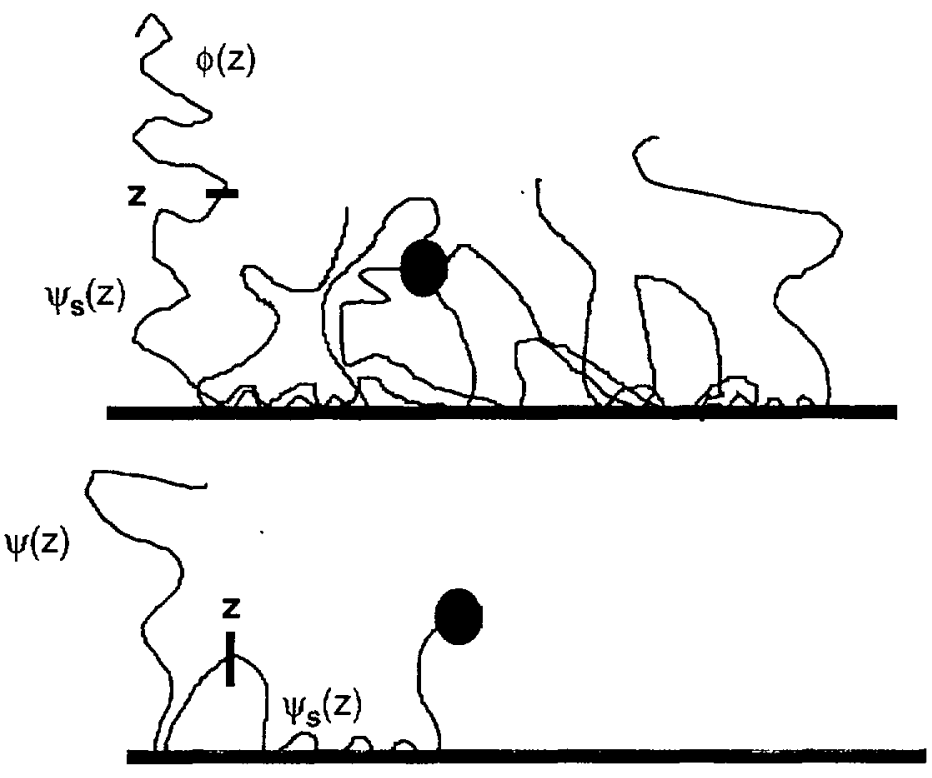

Fig. 3. - Order parameters for star polymer adsorption: $\phi(z)$ describes a tail dangling beyond $z$, $\psi_{\mathrm{s}}(z)$ describes a star with a branch ending at position $z, \psi(z)$ describes an adsorbed chain with an end point at position $z$. The loop monomer concentration carries a factor $\psi_{\mathrm{s}}(z) \psi(z)$ (see bottom), whereas the tail monomer concentration carries a factor $\psi_{\mathrm{s}}(z) \phi(z)$ (see top).

can be neglected. As for linear chains, in the limit of infinite molecular weights, $p \rightarrow 0$, the adsorbed star partition function can be written as $\tilde{Z}_{p}^{\text {sa }}(z)=\psi^{\mathbf{s}}(z) / p$ since the statistics of each arm is dominated by the bound state. Equation (10) for the partition function $\tilde{Z}_{p}^{\text {sa }}(z)$ imposes that

$$
p Z_{n=0}^{\mathrm{sa}}(z)=-\frac{a^{2}}{6} \frac{\partial^{2} \psi^{\mathrm{s}}}{\partial z^{2}}+U(z) \psi^{\mathrm{s}}+\varepsilon \psi^{\mathrm{s}}
$$

In the limit where the number of monomers in the selected arm vanishes $n=0$, the partition function $Z_{n=0}^{\mathrm{sa}}(z)$ is the partition function of a star of $(f-1)$ arms of $N$ monomers with its core (central point) at position $z$. It can be approximated by $Z_{n=0}^{\text {sa }}(z)=\psi^{(f-1)}(z)$. One can then check explicitly that the left hand side of equation (10) is negligible. The two order parameters $\psi$ and $\psi^{\text {s }}$ are therefore solutions of the same linear differential equation with the same boundary condition ( $\psi \rightarrow 0$ at infinity) they are thus proportional. We define the proportionality constant $k$ so that

$$
\psi=k \psi^{\mathrm{s}}
$$

The concentration inside the layer of adsorbed stars can be expressed as a function of the two partition functions $Z_{n}$ and $Z_{n}^{\mathrm{s}}$

$$
c(z) \sim \int \mathrm{d} n Z_{n}(z) Z_{N-n}^{\mathrm{s}}(z)
$$

It is written as a function of the order parameters as (see Fig. 3)

$$
v c(z)=\alpha^{\mathrm{s}}\left(\psi \psi^{\mathrm{s}}+\frac{1}{N} \phi \psi^{\mathrm{s}}\right)
$$


where $\alpha^{s}$ is a proportionality constant.

We now introduce an effective constant $\alpha=k \alpha^{s}$ and an effective number of monomers $N^{\prime}=2 N \alpha$, so that $U(z)=\alpha\left(\psi^{\mathrm{s} 2}+\frac{2}{N} \phi \psi^{\mathrm{s}}\right)$ the equations governing the order parameters $\psi^{\mathrm{s}}$ and $\phi$ for the adsorption of a dilute solution of star polymers are the same as the equations governing the adsorption of a dilute solution of linear chain with $N^{\prime}$ monomers and a proportionality constant $\alpha$ in equation (12).

Inside the adsorbed layer where the binding energy $\varepsilon$ is negligible, there is thus one characteristic length scale $z^{\times}$given by equation (13). The order parameters and the concentration. have the self-similar form of equations $(14,15)$ with $N$ replaced by $N^{\prime}$ and with the same functions $\tilde{\psi}(y), \tilde{\phi}(y)$ and $\tilde{c}(y)$.

The concentration of end points is $c_{\mathrm{e}}(z)=\frac{\alpha^{s}}{N} \psi_{s}(z)$ and the concentration of the central points (cores) is $c_{\mathrm{c}}(z)=\frac{\alpha^{\mathrm{s}}}{f N} \psi^{f}(z)$. The conservations of the end points and of the central points determine the adsorbance as

$$
v \Gamma=\int v c(z) \mathrm{d} z=\alpha^{\mathrm{s}} \int \psi_{s}(z) \mathrm{d} z=\alpha^{\mathrm{s}} \int \psi^{\mathrm{f}}(z) \mathrm{d} z
$$

This allows the determination of the two constants $\alpha^{s}$ and $k$

$$
\alpha^{\mathrm{s}}=\frac{a^{2}(f-1)}{3 d^{2} \log ^{f}\left(z^{*} / d\right)}, k=\frac{(f-1)}{\log ^{f-2}\left(z^{*} / d\right)}
$$

The crossover length $z^{2}$ between the regions dominated by loops close to the surface and by tails away from the surface is then

$$
z^{*}=\left[\frac{N a^{2} d}{62^{1 / 2}} \log \left(z^{*} / d\right)\right]^{1 / 3}
$$

It is important to notice that this is the same crossover length that would be obtained for the adsorption of linear chains of $2 \mathrm{~N}$ monomers. The concentration profiles (total concentration, loop concentration and end point concentration) inside the adsorbed layer are thus the same for star polymers made of $f$ arms of $N$ monomers and for linear chains of $2 N$ monomers. Qualitatively this means that if one starts from an adsorbed layer of linear chains of $2 \mathrm{~N}$ monomers and then crosslink them at their midpoint in order to form the stars the crosslinking process does not affect the concentration profiles.

The concentration of the star cores varies as

$$
v c_{\mathrm{c}}=\frac{\alpha^{s}}{f N^{f+1}} z^{\star 2 f} \tilde{\psi}^{f}\left(z / \tilde{z}^{\star}\right)
$$

It decays steeply as $1 /\left(N z^{f}\right)$ and all the end points are located in the very vicinity of the adsorbing surface.

As for the linear chain, the binding energy of the star $\varepsilon$ is found by balancing the chemical potentials of the adsorbed and free stars with a bulk monomer concentration $c_{\mathrm{b}}$. The partition function of an adsorbed star is $Z=\int \psi_{s}(z) \mathrm{d} z=\Gamma v / \alpha^{s}$ and we obtain

$$
\varepsilon=-\frac{1}{f N} \log \left(v c_{\mathrm{b}} / \alpha^{\mathrm{s}}\right)
$$

The constant $\alpha^{\text {s }}$ decreases with the number of arms $f$, therefore the binding energy is smaller for a star polymer than for a linear polymer with the same number of monomers. The thickness 
of the adsorbed layer $\lambda=a /(6 \varepsilon)^{1 / 2}$ is thus larger for a star polymer than for a linear polymer of the same number of monomers. Note however that this result is valid only at very low concentration when the adsorbed layer thickness is smaller than the radius of gyration of one arm $\sim N^{1 / 2} a$. At higher concentration, the thickness of the adsorbed layer of star polymers increases and saturates at the radius of gyration of the arm. The thickness of the adsorbed layer of linear polymer increases up to the radius of gyration of the linear chain $(f N)^{1 / 2} a$ and can eventually become larger than that of the star.

3.3. Adsorption of Comblike Polymers. - - Our second example of branched polymer is a comblike polymer. The backbone of the polymer is made of $k$ segments each containing $N_{\mathrm{b}}$ monomers; the comb has $(k-1)$ side chains each containing $N_{\mathrm{t}}$ monomers; the total number of monomers is $N=k N_{\mathrm{b}}+(k-1) N_{\mathrm{t}}$. As for the star polymer, we assume that the concentration is dilute enough that the thickness of the adsorbed layer is smaller than both the radii of gyration of the backbone segments $N_{\mathrm{b}}^{1 / 2} a$ and the side chains $N_{t}^{1 / 2} a$. This corresponds to a large enough binding energy of the polymer $\varepsilon N_{\mathrm{t}} \gg 1, \varepsilon N_{\mathrm{b}} \gg 1$. The study of the adsorption of a very dilute comb solution requires the determination of several partition functions. The partition function of a linear chain is as before split into an adsorbed chain contribution $\psi$ and a free chain contribution characterized by the order parameter $\phi$. These two order parameters satisfy equation (11) in the molecular potential created by the adsorbed combs. All the other partition functions are dominated by the adsorbed chains and the free chain contribution can be neglected. For any end point, we define the partition function of the polymer with the end point at a position $z, \psi_{\mathrm{e}}(z)$. We also define the partition function $\psi_{\imath}(z)$ of a comblike polymer with $i$ backbone segments and $(i-1)$ side chains with the constraint that one of the end points of the backbone is at position $z$ (note that $\psi_{1}(z)$ is the partition function of an adsorbed linear chain, $\psi(z)=\psi_{1}(z)$ ). All these partition functions are dominated by adsorbed states and do not depend on the molecular weights of the backbone segments and of the side chains. They all are solutions of the same Edwards equation

$$
0=-\frac{a^{2}}{6} \frac{\partial^{2} \psi}{\partial z^{2}}+U(z) \psi+\varepsilon \psi
$$

The boundary condition being the same for all these partition functions, they are all proportional to one another. We define the proportionality constant $k_{\imath}$ such that

$$
\psi_{2}(z)=k_{2} \psi_{k}(z)
$$

The total partition function of adsorbed comblike polymer is $Z=\int \psi_{\mathrm{e}}(z) \mathrm{d} z$ for any end point e. This imposes that the partition functions $\psi_{\mathrm{e}}(z)$ are identical for all of the end points (and in particular, $\psi_{\mathrm{e}}(z)=\psi_{k}(z)$ ). The partition function $Z$ can also be calculated by cutting the chains into two halves at any point of the backbone. We then obtain

$$
Z=\int \psi_{\mathrm{e}}(z) \mathrm{d} z=\int \psi_{\imath}(z) \psi_{k+1-\imath}(z) \mathrm{d} z=\int \psi(z) \psi_{\imath}(z) \psi_{k-\imath}(z) \mathrm{d} \tilde{z}
$$

In the vicinity of the adsorbing surface the concentration is dominated by the small loops and the concentration profile does not depend on the architecture of the polymer. We can thus anticipate that for any $i, v_{2}(z) \sim 1 / z$. Equation $(29)$ imposes then the value of the constants $k_{2}$

$$
k_{\imath}=\left[2 / \log \left(\tilde{z}^{\times} / d\right)\right]^{k-\imath}
$$

where $d$ is the adsorption length and $z^{*}$ the crossover distance between the loops and tails dominated regions defined below. 


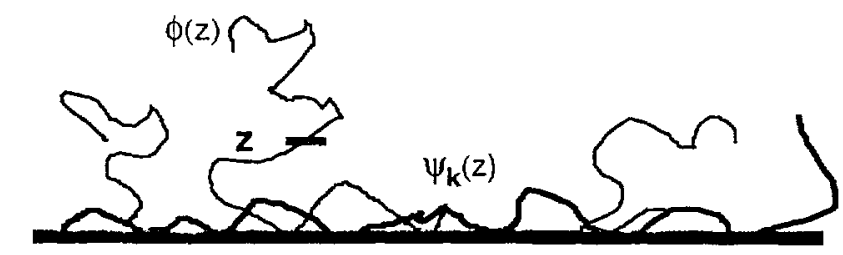

$\psi(z)$

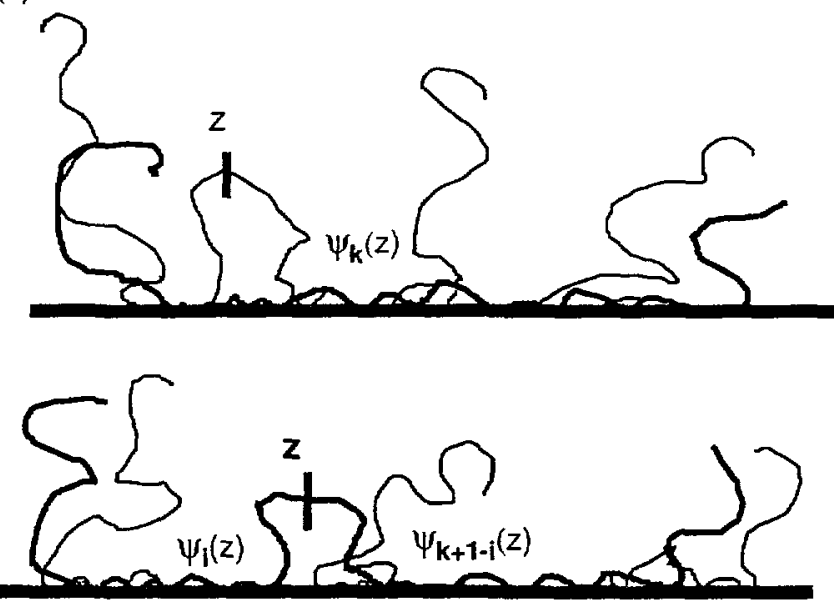

Fig. 4. - Order parameters for the adsorption of a comb polymer with $k$ backbone segments: $\phi(z)$ describes a tail dangling beyond $z, \psi_{\imath}(z)$ describes an adsorbed comb polymer comprising $i$ backbone segments with one end at position $z, \psi(z)$ describes a linear adsorbed chain with an end point at position $z$. The backbone loop monomer concentration carries a factor $\psi_{\imath}(z) \psi_{k+1-\imath}(z)$ summed over $i$ (see bottom), the side chain loop monomer concentration carries a factor $\psi(z) \psi_{k}(z)$ (see middle) whereas the tail monomer concentration carries a factor $\psi_{k}(z) \phi(z)$ (see top).

The molecular potential $U(z)$ can be expressed in terms of all the partition functions $\psi_{2}(z)$ as (see Fig. 4)

$$
U(z)=v c(z)=\frac{\alpha^{\mathrm{c}}}{N}\left[(k-1) N_{\mathrm{t}} \psi \psi_{k}+N_{\mathrm{b}} \sum_{\imath=1}^{k} \psi_{\imath} \psi_{k-\imath+1}+(k+1) \phi \psi_{k}\right]
$$

As for the star polymers, we introduce an effective constant $\alpha=\alpha^{\mathrm{c}}\left[2 / \log \left(z^{*} / d\right)\right]^{k-1}$ and an effective number of monomers $N^{\prime}$ defined by $\frac{2}{N^{\prime}}=\frac{k+1}{N}\left[\frac{\log \left(z^{*} / d\right)}{2}\right]^{k-1}$ Using the explicit values of the proportionality constants $k_{2}$, we write the molecular potential as $U(z)=$ $\alpha\left(\psi_{k}^{2}+\frac{2}{N} \phi \psi_{k}\right)$

The equations for the order parameters $\phi$ and $\psi_{k}$ are the same as for linear polymer adsorption and here also we can write inside the layer, the concentration in the self-similar form given by equations $(14,15)$. The constant $\alpha^{c}$ is obtained from the conservation of the end points, $\Gamma=\alpha^{c} \int \psi_{k} \mathrm{~d} z$

$$
\alpha^{c}=\frac{a^{2} 2^{k-1}}{3 d^{2} \log ^{k+1}\left(z^{*} / d\right)}
$$


The crossover length $z^{*}$ is here equal to

$$
z^{*}=\left[\frac{N a^{2} d}{62^{1 / 2}(k+1)} \log \left(z^{*} / d\right)\right]^{1 / 3}
$$

This is the same value that would be obtained for the adsorption of a linear chain with an effective number of monomers $N_{\text {eff }}=\frac{2 N}{k+1} \quad$ A simple way to understand this result is the following. We assume first that for these branched polymers, the concentration profiles are the same as for a neutral polymer with a crossover length $z^{*}$ corresponding to that of a linear polymer of $N_{\text {eff }}$ monomers. The concentration of end points is then written in a self-similar form as $v c_{\mathrm{e}}=\left(a^{2} / 6 z^{\times 2}\right)^{2} \tilde{\psi}(y)$. By integration over the coordinate $z$, we obtain the total number of end points as $2 \Gamma / N_{\text {eff }}$ where $\Gamma$ is the adsorbance (this is the same number of end points as for the equivalent linear chain). If the branched chain has $q$ end points, the total number of end points is $\Gamma q / N$ and $N_{\text {eff }}=2 N / q$ in agreement with our results both for starlike and comblike polymers.

The binding energy of the comblike polymers is obtained by balancing the chemical potentials of the adsorbed and free chains

$$
\varepsilon=-\frac{1}{N} \log \left(v c_{\mathrm{b}} / \alpha^{\mathrm{c}}\right)
$$

As for the star polymer, the binding energy is smaller than that of a linear chain of the same molecular weight and the thickness of the adsorbed layer $\lambda=a /(6 \varepsilon)^{1 / 2}$ is larger for the comblike polymer. Note however that these results are valid only at very low concentration where the thickness of the adsorbed layer is smaller than the radius of gyration of both the side chains and the backbone segments. As long as this condition remains valid, our results for the concentration profile remain valid even if all the backbone segments or all the side chains do not have the same number of monomers.

\section{Copolymer Adsorption}

4.1. Concentration of Adsorbed Monomers. - Our last example is the adsorption of a linear block copolymer. The copolymer is composed of a very long block A containing $N$ monomers which adsorbs on the interface and a small block $\mathrm{B}$ repelled by the interface. For simplicity, we assume that the $\mathrm{A}$ - and B-monomers are identical and have a different interaction with the wall. The junction point of the copolymer plays the role of one of the end points of the adsorbing block $A$ and is depleted from the surface. In the limit where the number $P$ of monomers of the B-block is small enough, the contribution of the B-monomers to the concentration profiles is negligible. We then require 4 order parameters to describe the adsorption of the copolymer. The partition function of a pure A chain of $N$ monomers is characterised by the two order parameters $\psi$ and $\phi$ which satisfy equation (11). We also consider the partition function of an A-B copolymer chain containing $n$ A-monomers and $P$ B-monomers with the end point of the A-block at position $z$. The contribution of the adsorbed states to this partition function defines an order parameter $\psi_{1}$ and the contribution of the free states an order parameter $\phi_{1}$. Using the procedure described in Section 3, these two order parameters are shown to obey the following equations

$$
\begin{aligned}
0 & =-\frac{a^{2}}{6} \frac{\partial^{2} \psi_{1}}{\partial z^{2}}+U(z) \psi_{1}+\varepsilon \psi_{1} \\
Z_{P}(z) & =-\frac{a^{2}}{6} \frac{\partial^{2} \phi_{1}}{\partial z^{2}}+U(z) \phi_{1}+\varepsilon \phi_{1}
\end{aligned}
$$



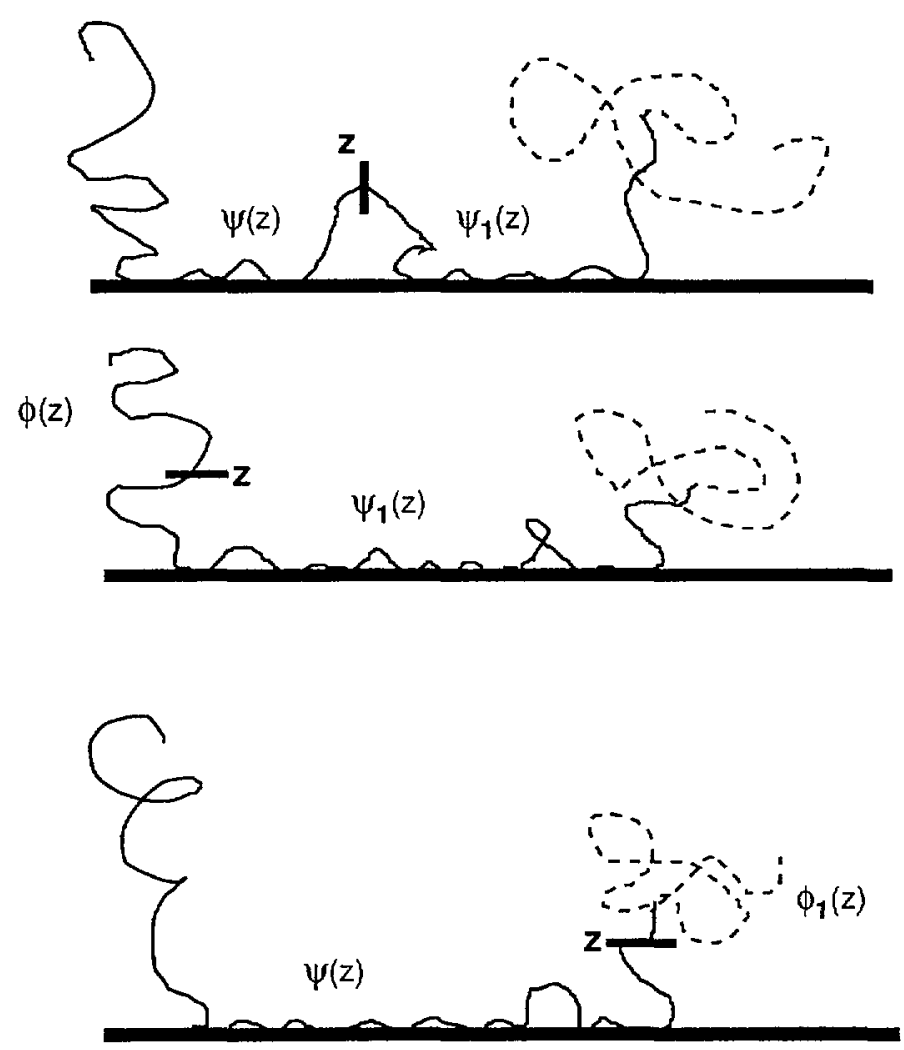

Fig. 5. - Order parameters for the adsorption of a block copolymer comprising a large adsorbing A-block and a small repelled B-block (dashed line): $\phi(z)$ describes the pure A tail dangling beyond $z, \psi(z)$ describes an adsorbed $\mathrm{A}$ chain with an end point at position $z, \psi_{1}(z)$ describes an adsorbed A B copolymer with its free $\mathrm{A}$ end at position $z, \phi_{1}(z)$ describes the tail comprising the $\mathrm{B}$ block dangling beyond $z$. The A loop monomer concentration carries a factor $\psi(z) \psi_{1}(z)$ (see top), the A tail monomer concentration carries a factor $\phi(z) \psi_{1}(z)$ (see middle), the A tail monomer concentration on the B block side carries a factor $\psi(z) \phi_{1}(z)$ (see bottom).

where $Z_{P}(z)$ is the partition function of a B-chain of $P$ monomers with one end point at position $z$. We assume in the following that $B$-chains are small enough that the radius $R_{P}=P^{1 / 2} a / 6^{1 / 2}$ is smaller than any characteristic length scale of the profile and in particular that it is smaller than the crossover length $z^{\times}$between the regions dominated by loops and tails. In this limit, the partition function $Z_{P}(z)$ is small if $z<R_{P}$ and is equal to 1 if $z \gg R_{P}$. In the adsorbed layer, the concentration is dominated by the A-monomers and the molecular potential can be written as (see Fig. 5)

$$
U(z)=\alpha^{c}\left(\psi \psi_{1}+\frac{\phi \psi_{1}}{N}+\frac{\phi_{1} \psi_{1}}{N}\right)
$$

Over most of the adsorbed layer, (except within a thin layer of thickness $R_{P}$ in the vicinity of the adsorbing surface), the two functions $\phi$ and $\phi_{1}$ are equal. As before the two functions $\psi$ and $\psi_{1}$ are proportional and we write $\psi=k \psi_{1}$; as in our previous examples, we can also anticipate that these functions are proportional to $1 / z$. The partition function of the adsorbed 
copolymer can be written as $Z=\int \mathrm{d} z \psi_{1}(z)=\int \psi(z) Z_{P}(z) \mathrm{d} z$. This imposes that the constant $k$ is approximately given by $k=\log \left(z^{\times} / d\right) / \log \left(z^{\times} / R_{P}\right)$. The constant $\alpha^{\mathrm{c}}$ is obtained from the conservation of the end points $v \Gamma=\int U(z) \mathrm{d} z=\alpha^{\mathrm{c}} \int \mathrm{d} z \psi_{1}(z)$ and

$$
\alpha^{c}=\frac{a^{2}}{3 d^{2} \log \left(z^{*} / d\right) \log \left(z^{*} / R_{P}\right)}
$$

Inside the adsorbed layer, the concentration profile is the same as that of linear homopolymer chains with an effective constant $\alpha=k \alpha^{\mathrm{c}}$ and an effective number of monomers $N^{\prime}=2 \mathrm{Nk} /(1+$ $k)$. The crossover length between the loop and tail regions is then

$$
z^{*}=\left[\frac{N a^{2} d}{62^{1 / 2}\left[\log \left(z^{*} / d\right)+\log \left(z^{*} / R_{P}\right)\right]} \log \left(z^{*} / d\right) \log \left(z^{*} / R_{P}\right)\right]^{1 / 3}
$$

The crossover length is thus smaller for the copolymer than for the pure homopolymer. By writing the chemical potential balance between adsorbed and free copolymer chain, we obtain the binding energy as $\varepsilon=-(1 / N) \log \left(v c_{\mathrm{b}} / \alpha^{\mathrm{c}}\right)$. The constant $\alpha^{\mathrm{c}}$ is larger for the copolymer and $\varepsilon$ is larger for the copolymer than for the pure A homopolymer. The thickness of the layer is thus smaller for the copolymer.

4.2. Concentration of the Non-Adsorbed Block. - The determination of the concentration of the $\mathrm{B}$-monomers requires the determination of two partition functions: the partition function $Z_{n}(z)$ of a $\mathrm{B}$-chain of $n$ monomers with one end point at position $z$ in the external potential $U(z)$ (created by the adsorbed A-monomers) and the partition function $Z_{n}^{1}(z)$ of a copolymer chain comprising a B-block of $n$ monomers and an A-block of $N$ monomers with the end point of the B-block at position $z$. As above, we determine the Laplace transforms of these partition functions $\tilde{Z}_{p}$ and $\tilde{Z}_{p}^{1}$. They satisfy the following equations which are directly derived from (9)

$$
\begin{aligned}
-p \tilde{Z}_{p}+1 & =-\frac{a^{2}}{6} \frac{\partial^{2} Z_{p}}{\partial z^{2}}+U(z) \tilde{Z}_{p} \\
-p \tilde{Z}_{p}^{1}+\psi(z) & =-\frac{a^{2}}{6} \frac{\partial^{2} \tilde{Z}_{p}^{1}}{\partial z^{2}}+U(z) \tilde{Z}_{p}^{1}
\end{aligned}
$$

The Laplace transform of the concentration with respect to the number of monomers $P$ of the B-block is then $v \tilde{c}(z)=\frac{\alpha^{c}}{N} \tilde{Z}_{p}(z) \tilde{Z}_{p}^{1}(z)$. The boundary condition for the non adsorbing B-chains is that the partition functions $\tilde{Z}_{p}$ and $\tilde{Z}_{p}^{1}$ vanish at a distance $z=d$ from the interface. Equation (38) can then be solved. We use here $a / 6^{1 / 2}$ as the unit length and consider only distances larger than the monomer size $z \gg d$; we obtain

$$
\begin{aligned}
\tilde{Z}_{p}^{1}= & \frac{\psi(z)}{p}\left[1-\mathrm{e}^{-\omega z}(1+w z)\right] \\
\tilde{Z}_{p}= & -\frac{1}{2 p}\left\{-2+\left[\mathrm{e}^{-\omega z}\left(\frac{1}{z}+\omega\right)-\mathrm{e}^{\omega z}\left(\frac{1}{z}-\omega\right)\right]\left[\frac{\log z}{\omega}-\int_{0}^{\infty} \mathrm{e}^{\left.-\omega z^{\prime} \log z^{\prime} \mathrm{d} z^{\prime}\right]}\right.\right. \\
& -\left[\mathrm{e}^{-\omega z}\left(\frac{1}{z}+\omega\right) \int_{0}^{z} \mathrm{e}^{\omega z^{\prime}} \log \left(z^{\prime} / z\right) \mathrm{d} z^{\prime}-\mathrm{e}^{\omega z}\left(\frac{1}{z}-\omega\right) \int_{0}^{z} \mathrm{e}^{-\omega z^{\prime}} \log \left(z^{\prime} / z\right) \mathrm{d} z^{\prime}\right]
\end{aligned}
$$

where $\omega^{2}=p$. These expressions are valid when $z \ll z^{\star}$. In this regime, we have used the fact that $\psi(z) \sim 1 / z$. 
In the limit where $z$ is large (compared to the radius of gyration $R_{P}$ of the B-block), $\tilde{Z}_{p}^{1}=$ $\psi(z) / p$ and $\tilde{Z}_{p}=1 / p$; the two partition functions are thus $Z_{n}^{1}(z)=\psi(z)$ and $Z_{n}(z)=1$ as expected. In the limit where $z$ is small, the two partition functions are $\tilde{Z}_{p}^{1}=\psi(z)\left(z^{2} / 2-\right.$ $\left.\omega z^{3} / 3 \omega\right)$ and $\tilde{Z}_{p}=-\left(z^{2} / 3\right) \log (\omega z / A)$ where $A=\int_{0}^{\infty} \mathrm{e}^{-t} \log t \mathrm{~d} t+\frac{4}{3}$ The B monomers are depleted from the adsorbing surface, and the two partition functions vanish in this limit. The partition function $Z_{n}^{1}(z)$ vanishes as $Z_{n}^{1}(z)=\psi(z) \frac{z^{3}}{6 \pi^{1 / 2} n^{3 / 2}}$, it is proportional to $z^{2}$ in the vicinity of the interface. The partition function of a homopolymer $\mathrm{B}$ chain $Z_{n}(z)$ has the same behavior as the order parameter $\phi$ as could be expected. If we introduce the concentration of the junction point $v c_{\mathrm{j}}=\left(\alpha^{\mathrm{c}} / N\right) \psi(z)$ the concentration of $\mathrm{B}$ monomers has the following asymptotic behaviors

$$
\begin{aligned}
& v c_{\mathrm{B}}(z)=v c_{\mathrm{j}}(z) \frac{3 z^{4}}{P a^{4}} \quad\left(z<R_{P}\right) \\
& v c_{\mathrm{B}}(z)=v c_{\mathrm{j}}(z) P \quad\left(z>R_{P}\right)
\end{aligned}
$$

where we have reestablished the original length unit. In the vicinity of the wall, the B-monomers are depleted and their concentration increases as $z^{3}$. The concentration reaches a maximum at a distance from the wall of the order of the radius of of the $B$ block $R_{P} \sim P^{1 / 2} a$ and then decreases as $c_{\mathrm{j}}(z)$ i.e. as $1 / z$ if $z<z^{*}$ and as $1 / z^{4}$ if $z \gg z^{*}$.

\section{Concluding Remarks}

The aim of this paper was to study the effect of the polymer architecture on the distributions of tails and loops in adsorbed polymer layers.

Adsorbed ring polymer layers obviously contain no tails and can be essentially described by the standard mean field theory based on the so-called ground state dominance approximation, we have explicitly calculated the corrections to this approximation and checked that they remain small.

For all the other architectures studied (branched polymers, copolymers) the concentration profile is the same as for homopolymers both at short length scales $\left(c(z)=a^{2} /\left(3 z^{2}\right)\right)$ and at large length scales $\left(c(z)=10 a^{2} /\left(3 z^{2}\right)\right)$. The architecture of the polymer is only important in the crossover between the loop and tail regions and for the overall thickness of the adsorbed layer $\lambda$.

One of the main limitations of our approach is that we use a mean field model which ignores the excluded volume correlations. These could be of major importance for example for star polymers containing many arms: in the vicinity of the center of the star, the chains are strongly stretched and the elasticity of the star depends on this stretching which is ignored by our mean field theory. A scaling theory for stars in this limit has been constructed in reference [14] but it does not study in details the statistics of loops and tails. For branched polymers with a low functionality, the mean field theory is expected to give a good qualitative description of adsorption. Another important limitation of our study is that we have only considered branched polymers where all the branches are larger than the adsorbed layer thickness. It seems difficult to extend the theory to polymers containing a large number of small branches. For copolymer adsorption, we have only considered very short repelled blocks; in the limit where the repelled block becomes larger than the adsorbed layer thickness, the adsorbed copolymer layer has a double layer structure with an inner layer formed by the adsorbed A monomers and a brushlike structure formed by the B-monomers discussed in reference [15]. It would be 
interesting to study the crossover towards this regime as the length of the B-block is increased. Because of these limitations we have not attempted any comparison with experimental results. A direct comparison could also be possible with the results obtained from the Scheutjens-Fleer approach [16].

Several extensions to this work are possible. The most interesting ones seem the influence of the bulk concentration of the adsorbed polymer and the adsorption competition between polymers of different architectures.

\section{References}

[1] Fleer G., Cohen-Stuart M., Scheutjens J., Cosgrove T. and Vincent B., Polymers at interfaces (Chapman and Hall, London, 1993).

[2] Eisenriegler E., Polymers near surfaces (World Scientific Singapore, 1993) .

[3] Scheutjens J., PhD Thesis, Agricultural University Wageningen (1978).

[4] de Gennes P.G., Ad. Colloid Inter. Sci. 27 (1987) 189.

[5] Semenov A.N. and Joanny J.F., Europhys. Lett. 29 (1995) 279.

[6] Semenov A., Bonet-Avalos J., Johner A. and Joanny J.F., Macromolecules in press.

[7] Johner A., Bonet-Avalos J., Semenov A., Joanny J.F. and van der Linden C., submitted to Macromolecules (1995).

[8] de Gennes P.G., Scaling Concepts in Polymer Physics (Cornell University Press, Ithaca, 1985).

[9] van Lent B., Scheutjens J. and Cosgrove T., Macromolecules 20(1987) 366.

[10] Edwards S.F., Proc. Phys. Soc. 85(1965) 613.

[11] Johner A., Joanny J.F. and Rubinstein M., Europhys. Lett. 22 (1993) 591.

[12] The concentration profile of equation (5) is accurate if $y$ is not too small; if $y$ is small the dominant contribution to the concentration is due to the adsorbed chains and is well described by the ground state dominance approximation (the second term in (5)). The small contribution of the non adsorbed chains (the first term) requires a more rigorous inversion of the Laplace transform.

[13] Yamakawa H, Modern Theory of Polymer Solutions (Harper and Row, New York, 1971).

[14] Halperin A. and, Joanny J.F., J. Phys. II France 1 (1991) 623.

[15] Marques C. and Joanny J.F., Macromolecules 22(1989) 1464.

[16] van der Linden C., PhD Thesis, Agricultural University Wageningen (1995). 